\title{
RESEARCH NOTES
}

\section{ALMOST CONVEX METRICS AND PEANO COMPACTIFICATIONS}

\author{
R.F. DICKMAN, JR. \\ Department of Mathematics \\ Virginia Polytechnic Institute \\ and State University \\ Blacksburg, Virginia 24061 U.S.A. \\ (Received June 23, 1981)
}

ABSTRACT. Let $(\mathrm{X}, \mathrm{d})$ denote a locally connected, connected separable metric space. We say the $\mathrm{X}$ is S-metrizable provided there is a topologically equivalent metric $\rho$ on $\mathrm{X}$ such that $(\mathrm{X}, \rho)$ has Property $S$, i.e., for any $\varepsilon>0, X$ is the union of finitely many connected sets of $p$-diameter less than $\varepsilon$. It is well-known that S-metrizable spaces are locally connected and that if $\rho$ is a Property $S$ metric for $X$, then the usual metric completion $(\tilde{X}, \tilde{\rho})$ of $(X, \rho)$ is a compact, locally connected, connected metric space; i.e., $(\mathrm{X}, \rho)$ is a Peano compactification of $(\mathrm{X}, \rho)$. In an earlier paper, the author conjectured that if a space $(X, d)$ has a Peano compactification, then it must be S-metrizable. In this paper, that conjecture is shown to be false; however, the connected spaces which have Peano compactificatons are shown to be exactly those having a totally bounded, almost convex metric. Several related results are given. KEY (WORDS AND PHRASES. Almost Convex Metrics, Property $S$ metrics, Peano spaces, Compactifications.

1980 MATHEMATICS SUBJECT CLA.SIFICATICNS CODES. 54F25, 54E 35.

1. INTRODUCTION.

Throughout this note let $(X, d)$ denote a metric space. We say that $d$ is convex 
provided that, for any pair $x, y \in X$, there is $z \varepsilon X$ such that $d(x, z)=d(z, y)=d(x, y) / 2$. It is almost convex if, for $\mathrm{x}, \mathrm{y} \varepsilon \mathrm{X}$ and $\varepsilon>0$, there is $\mathrm{z} \varepsilon \mathrm{X}$ such that $|\mathrm{d}(\mathrm{x}, \mathrm{z})-\mathrm{d}(\mathrm{x}, \mathrm{y}) / 2|$ $<\varepsilon$ and $|\mathrm{d}(\mathrm{z}, \mathrm{y})-\mathrm{d}(\mathrm{x}, \mathrm{y}) / 2|<\varepsilon[1,2]$.

We say that $\mathrm{X}$ is S-metrizable provided there is a topologically equivalent metric $\rho$ on $X$ such that $(X, \rho)$ has Property $S$, i.e., for any $\varepsilon>0$, $X$ is the union of finitely many connected sets of $\rho$-diameter less than $\varepsilon$. It is we11-known that Smetrizable spaces are locally connected and that if $\rho$ is a Property $S$ metric for $X$, then the usual metric completion $(\tilde{X}, \tilde{\rho})$ of $(X, \rho)$ is a compact, locally connected, connected metric space, i.e., $(\tilde{X}, \tilde{\rho})$ is a Peano compactification of $(X, \rho)[3, p .154]$.

It is a famous result of R. H. Bing that any continuous curve $\mathrm{P}$ (i.e., a compact, locally connected, connected metric space) can be assigned a convex metric [1].

In an earlier paper [4], the author conjectured that, if $\mathrm{X}$ is locally connected and if $\mathrm{X}$ has a Peano compactification, then $\mathrm{X}$ is S-metrizable. In this paper we show, by example, that this conjecture is false; however, we do obtain a characterization of such spaces in terms of the existence of a totally bounded, almost convex metric for $X$. We also obtain several related results characterizing totally bounded (Smetrizable, almost convex) metrics.

\section{PEANO COMPACTIFICATIONS.}

THEOREM 2.1. A connected metric space $(\mathrm{X}, \mathrm{d})$ has a Peano compactification if and only if it has a topologically equivalent totally bounded, almost convex metric.

PROOF. The necessity. Let $(P, r)$ be a Peano compactification of $X$, i.e., $P$ is a continuous curve and $X$ is a dense subset of $P$. By $R$. H. Bing's result, there exists an equivalent metric $\rho$ for $P$ such that $\rho$ is convex. It then follows that $\sigma=f \mid X$ is totally bounded and almost convex; cf. [1, Thm. 10].

The Sufficiency. Let $\mathrm{r}$ be an almost convex, totally bounded metric for $\mathrm{x}$. Let $(\tilde{X}, \tilde{r})$ be the usual metric completion of $(X, r)$. We will argue that $(\tilde{X}, \tilde{r})$ is a Peano compactification of $(X, r)$. Clearly, $\tilde{X}$ is compact since $r$ is totally bounded. Furthermore, $\tilde{r}$ is a convex metric for $\tilde{X}$; let $x, y \varepsilon \tilde{x}$. Since $r$ is almost convex, there exists a sequences $x_{1}, x_{2}, \ldots, y_{1}, y_{2}, \ldots$, and $z_{1}, z_{2}, \ldots$ in $x$ such that

$$
\left|r\left(x_{n}, z_{n}\right)-r\left(x_{n}, y_{n}\right) / 2\right|<2^{-n} \text { and }\left|r\left(z_{n}, y_{n}\right)-r\left(x_{n}, y_{n}\right) / 2\right| \cdot 2^{-n} \text {. }
$$


Since $r$ is totally bounded, without loss of generality, we may assume that each of the sequences $x_{1}, x_{2}, \ldots, y_{1}, y_{2}, \ldots$, and $z_{1}, z_{2}, \ldots$ is Cauchy in $x$. Then by the completeness of $(\tilde{X}, \tilde{r})$, it follows that $\lim _{n \rightarrow \infty} x_{n}=x$ and $\lim _{n \rightarrow \infty} y_{n}=y$. Furthermore, if $\lim _{n \rightarrow \infty} z_{n}=z, \tilde{r}(x, z)=\tilde{r}(z, y)=\tilde{r}(x, y) / 2$ since $\tilde{r}$ is continuous. Thus $\tilde{r}$ is convex and complete. It follows from Theorem 3.1 of [5] that the spheres $\mathrm{S}_{\mathrm{r}}(\mathrm{x}, \varepsilon)$ of $\tilde{\mathrm{X}}$ are connected sets. This implies that $\tilde{X}$ is locally connected and this completes the proof. EXAMPLE 2.1. Let $\mathrm{P}$ be the square $\left\{(\mathrm{x}, \mathrm{y}) \in \mathbb{R}^{2}: 0 \leq \mathrm{x}, \mathrm{y} \leq 1\right\}$ in the plane. For $\mathrm{n} \in \mathbb{N}$, let $\mathrm{L}_{\mathrm{n}}=\{(1 / \mathrm{n}, \mathrm{y}): 0<\mathrm{y}<1\}$ and let $\mathrm{L}_{0}=\{(0, \mathrm{y}): 0<\mathrm{y}<1\}$. Set $\mathrm{x}=$ $\mathrm{P} \backslash_{\mathrm{n}=0}^{\stackrel{\infty}{\cup}} \mathrm{L}_{\mathrm{n}}$. Then $\mathrm{P}$ is a Peano compactification of $\mathrm{X}$; however, $\mathrm{X}$ is not S-metrizable. Suppose $\rho$ is an S-metric for $X$ and let $A=\{(x, 1): 0 \leq x \leq 1\}$ and $B=\{(x, 0): 0 \leq x \leq$ 1\}. Then $A$ and $B$ are compact and hence $\rho(A, B)=\varepsilon>0$. Now the components $C_{1}, C_{2}$, ... of $X \backslash(A \cup B)$ have limit points in each of $A$ and $B$. Thus, any collection of connected sets of $\rho$-diameter less than $\varepsilon / 3$ that covers a component $C_{n}$ has at least one such connected subset lying entirely in $C_{n}$. This implies that $\rho$ is rot an S-metric for $X$; however, if $d$ is the relative metric on $X$ inherited from the usual metric on $\mathrm{P}, \mathrm{d}$ is almost convex and totally bounded.

\section{RELATED RESULTS.}

A compatible normal sequence in a space $z$ is a sequence $U_{1}, U_{2}, \ldots$ of open covers of $Z$ such that $U_{n+1}$ star-refines $U_{n}$ for $n=1,2, \ldots$ and so, for any $x \in Z,\left\{\right.$ St $\left(x, U_{n}\right)$ : $\mathrm{n}=1,2, \ldots\}$ is a neighborhood base for $\mathrm{x}[5]$.

THEOREM 3.1. [6, Prop. 23.4] $\mathrm{A} \mathrm{T}_{0}$-space is metrizable if and only if it has a compatible normal sequence.

COROLLARY 3.1. A metric space $X$ is totally bounded if and only if $X$ has a compatible normal sequence $U_{1}, U_{2}, \ldots$ where each $U_{n}$ is a finite cover of $x$.

PROOF. Suppose $(\mathrm{X}, \mathrm{d})$ is totally bounded. It follows from the total boundedness of $(X, d)$ that there is a finite open cover $U_{1}$ of $X$ such that $\delta_{d}(U)=1 / 3$ for all Uह $U_{1}$ where $\delta_{d}(U)=\sup \{d(x, y): x, y \in U\}$, the d-diameter of $U$. Since $U_{1}$ is finite, there is a Lebesgue number $\varepsilon_{1}<3^{-2}$ such that, if $\mathrm{d}(\mathrm{x}, \mathrm{y})<\varepsilon_{1}$, then $\mathrm{x}$ and $\mathrm{y}$ be in some member of $U_{1}$. Again, by the total boundedness of $(x, d)$, there is a finite open cover $v_{1}$ of $x$ such that $\delta_{d}(V)<\varepsilon_{1}$. If $\varepsilon_{2}<\varepsilon_{1}$ is a Lebesgue number for $v_{1}$ and $U_{2}$ is any $f$ ini 
open cover of $X$ such that $\delta_{d}(U)<\varepsilon_{2}$ for any $U \varepsilon U_{2}$, then $U_{2}$ star-refines $U_{1}$. Continue in this manner and obtain a compatible normal sequence $u_{1}, u_{2}, \ldots$ for $x$.

On the other hand, suppose $u_{1}, u_{2}, \ldots$ is a compatible normal sequence for $\mathrm{X}$ where each $u_{n}$ is finite. Then, in the usual metric $\rho$ for $x$ that is associated with $u_{1}, u_{2}$, $\ldots$ as given by $s$. Willard $[6], \delta_{\rho}(U)<2^{n-1}$ and $U \varepsilon U_{n}, n=2,3, \ldots$. It then follows that, since each $U_{n}$ is finite, $\rho$ is a totally bounded metric for $x$. This completes the proof.

COROLLARY 3.2. A metric space $(X, d)$ is S-metrizable if and only if it has a compatible normal sequence $u_{1}, u_{2}, \ldots$ where each $u_{n}$ is a finite cover and the members of $U_{n}$ are connected sets.

PROOF. The necessity follows from the argument above, together with the observation that the covers $u_{1}, u_{2}, \ldots$ can be selected so as to consist of finitely many open and connected sets.

The sufficiency. We observe that, if $u_{1}, u_{2}, \ldots$ is a compatible normal sequence for $X$ where each $U_{n}$ is finite and the members of $U_{n}$ are connected sets and if $\rho$ is the usual metric associated with $U_{1}, U_{2}, \ldots$ as given in [6], then, for $U \varepsilon U_{n}, \delta_{\rho}(U)<$ $2^{n-1}, n=2,3, \ldots$ and the sets $U \varepsilon U_{n}$ are connected. Thus, for any $\varepsilon>0$ and $k \varepsilon \mathbb{N}$ so that $0<2^{-k}<\varepsilon, x=U\left\{U: U \varepsilon U_{k}\right\}$ is a finite cover of $x$ by connected sets of $\rho$-diameter less that $\varepsilon$. This completes the proof.

THEOREM $3.2[2]$. A connected metric space $X$ has an almost convex metric if and only if it has a compatible normal sequence $u_{1}, u_{2}, \ldots$ such that (i) each pair of points that is covered by either an element of $u_{n+1}$ or the union of a pair of intersecting elements of $u_{n+1}$ can be covered by an element of $u_{n}$ and (ii) each pair of points that can be covered by an element of $u_{n}$ can be covered by the union of two intersecting elements of $u_{n+1}$.

It is, apparently, very difficult to combine the total boundedness (finiteness) conditions of Corollaries 3.1 and 3.2 and the intersection-type properties of Theorem 3.2. It would te very desirable to do so in light of the results of the previous section. 


\section{REFERENCES}

1. BING, R.H. Partitioning a set, Bul1. Amer. Math. Soc. 55, 1101-1110, 1949.

2. BING, R.H. Extending a metric, Duke Math. J. 14, 511-519, 1947.

3. WHYBURN. G.T. Analytic Topology, Amer. Math. Soc. Colloq. Publ, Vol. $28,1942$.

4. DICKMAN, R.F., JR. Peano compactifications and Property S metric spaces, Internat. J. Math. and Math. Sci. 4, 695-700, 1980.

5. LELEK, A. and NITKA, W. On convex metric spacesI, Fund. Math. 49, 183-204, 1961.

6. WILLARD, S. Genera1 Topology, Addison-Wesley, 1968. 


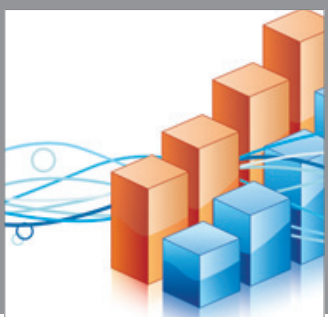

Advances in

Operations Research

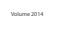

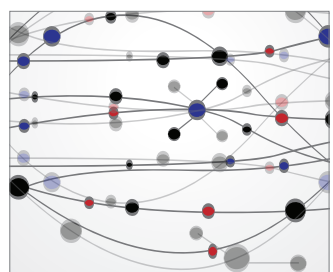

\section{The Scientific} World Journal
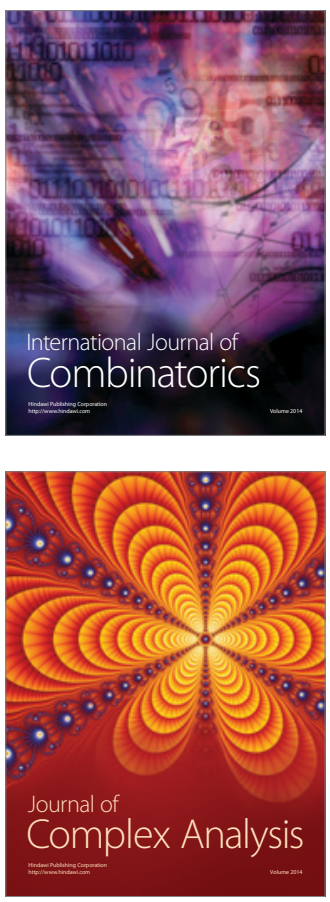

International Journal of

Mathematics and

Mathematical

Sciences
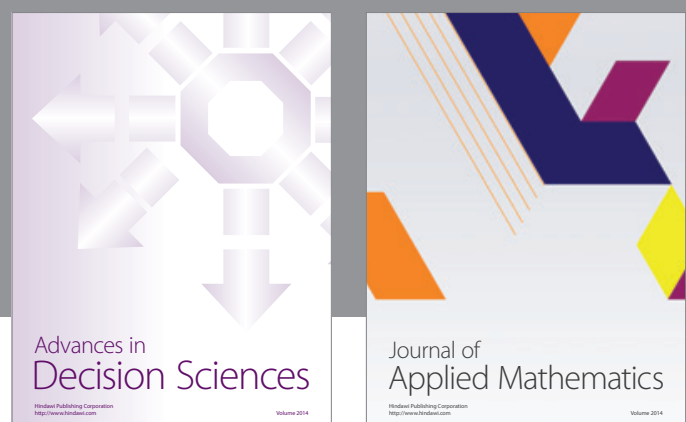

Journal of

Applied Mathematics
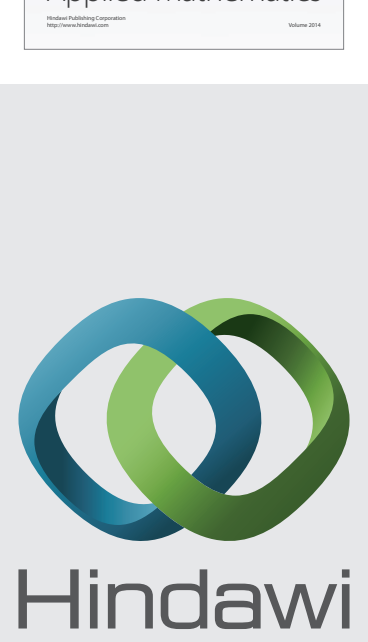

Submit your manuscripts at http://www.hindawi.com
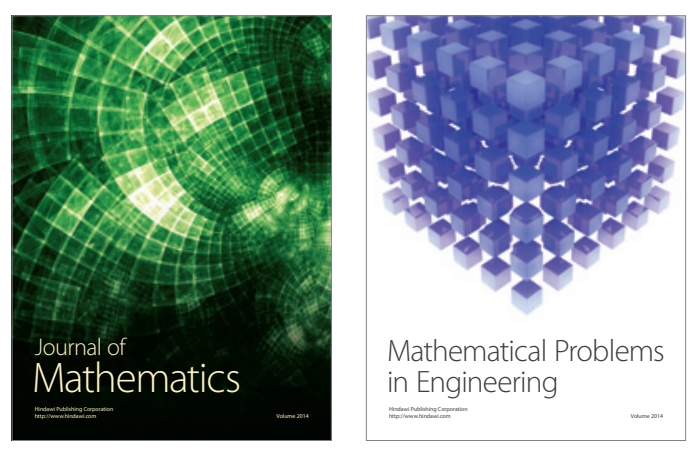

Mathematical Problems in Engineering
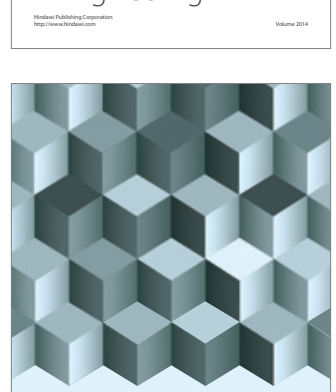

Journal of

Function Spaces
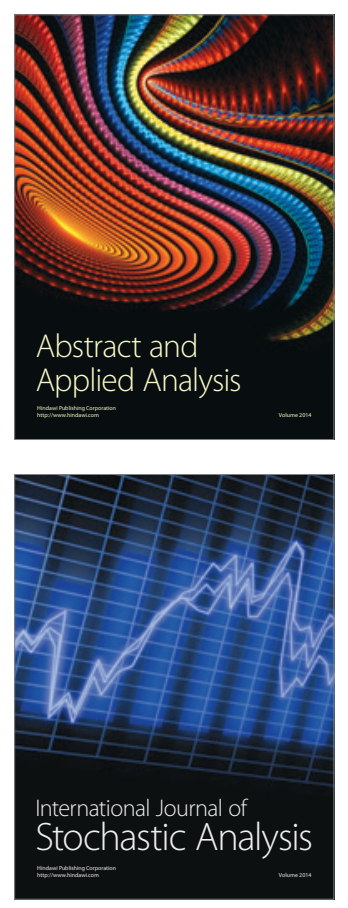

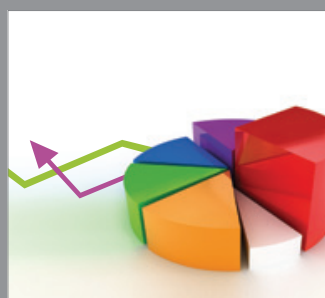

ournal of

Probability and Statistics

Promensencen
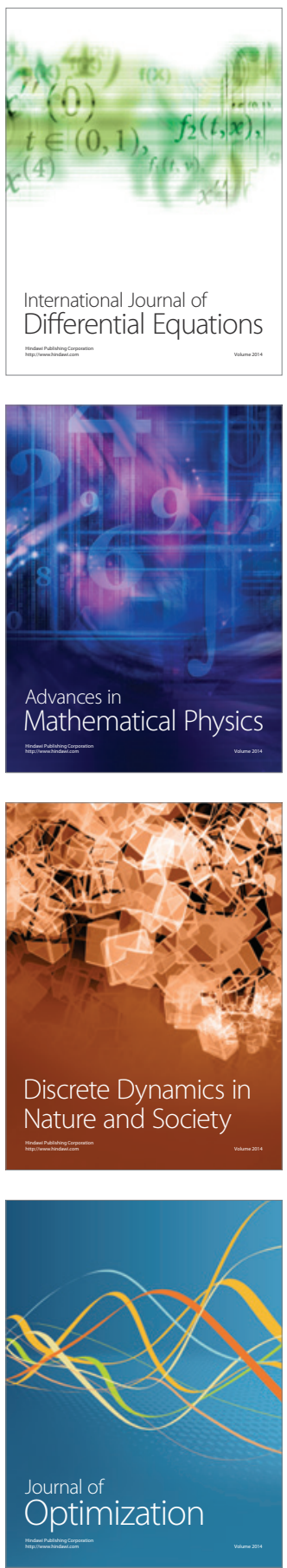